\title{
LA CÚPULA DE LA IGLESIA CARTUJANA DE ARA CHRISTI (EL PUIG, VALENCIA). GÉNESIS, EVOLUCIÓN Y ARTÍFICES DE SU CONSTRUCCIÓN
}

POR

\author{
ALBERT FERRER ORTS
}

\begin{abstract}
Se ofrece un pormenorizado relato de la construcción de la cúpula de la cartuja de Ara Christi, desde su concepción hasta las diversas reparaciones de las que fue objeto en el siglo XVII y se especifican los artífices de las diversas fases de su construcción.

Palabras clave: Ara Christi; arquitectura; siglo xvi; siglo XvII.

The author offers a detailed account of the construction of the cupola of the Carthusian monastery of Ara Cristi in El Puig (Valencia) from its conception in the sixteenth century through the various restorations it underwent in the seventeenth, and includes specific information on the artisans responsible for the distinct stages of construction.
\end{abstract}

Key words: Ara Christi; architecture; sixteenth century; seventeenth century.

Hace unos años, a propósito de la sombra proyectada por la cúpula de El Escorial —es decir, su estela en la arquitectura española de época moderna-, los profesores Bustamante y Marías ofrecían una completa visión de su génesis y posterior evolución, llegando a la conclusión de que su desarrollo obedecía básicamente a la hipervaloración de la antigüedad clásica (perfectamente plasmada en el Panteón de Adriano en Roma) mediante la conjugación de dos factores concatenados: por un lado, su dependencia de modelos propios del renacimiento italiano y, por otro, su vinculación con la simbología funeraria ${ }^{1}$.

Con todo, lo que más nos llamó la atención de su elaborada disertación fue la temprana asimilación que de ella se hizo en el medio constructivo valenciano - como ya señalara $F$. Benito-, claramente supeditado en las postrimerías del s. XVI a patrones ligados a la tradición gótica $^{2}$. Así lo atestiguan la cúpula de la iglesia del Colegio del Corpus Christi (concluida hacia 1597), la correspondiente a la capilla de la Comunión del convento del Carmen (construida sobre 1613), ambas de Valencia; la de la iglesia de la cartuja de Ara Christi (objeto de

\footnotetext{
1 Bustamante, A.-Marías, F. «La sombra de la cúpula de El Escorial», Fragmentos, 4-5, 1985, pp. 46-63.

${ }^{2}$ Como lo atestiguan la propia iglesia del Patriarca, alzada sobre amplios tramos abovedados con crucería simple por Guillem del Rey entre 1590-1594 fundamentalmente, o el templo de la misma cartuja de Ara Christi, construido a partir de 1621, donde se complementan bóvedas de crucería estrellada con otras simples.
} 
este estudio) o la perteneciente al templo jerónimo de San Miguel de los Reyes (rematada en 1644) ${ }^{3}$.

Sin embargo, otros ejemplares como la cúpula de la iglesia de la Compañía de Jesús (finalizada en 1631) ${ }^{4}$ o la perteneciente al templo de la Universidad de Gandia (1634-1636) ${ }^{5}$, no fueron contemplados en aquella ocasión.

Nuestra intención, no obstante, no es la de dilucidar hasta qué punto cuajaron las novedades en forma de cúpula extradosada sobre tambor en el ámbito valenciano, añadiendo a la lista conocida nuevos ejemplares estudiados desde entonces, como tampoco polemizar con un texto tan bien trabado como el de este fructífero binomio de autores. Más bien, es nuestra voluntad ofrecer un pormenorizado relato de la erección de la cúpula cartujana de Ara Christi, desde su concepción hasta las diversas reparaciones de las que fue objeto a lo largo del s. XVII, haciendo hincapié en los artífices que participaron en las diversas fases de su construcción, y con ello contribuir a un mejor conocimiento de esta peculiar tipología edilícia en sus inicios.

\section{La Cartuja de ARa Christi (1584-1621)}

El monasterio del Puig fue la cuarta fundación de la orden de San Bruno en el reino de Valencia ${ }^{6}$. Tiene su origen en la voluntad de doña Elena Roig y de Artés de legar una parte de sus heredades (la alquería «vulgarment dita de mossén Roig») ${ }^{7}$ a la Cartuja, con la condición de que «los pares que la fundaran dita casa de Nostra Señora de Arachristi no sien subiectes a ningun convent de dita cartoxa» ${ }^{8}$.

El 2 de noviembre de 1582 doña Elena Roig, ante el notario Jaume Cristòfor Ferrer y tres clérigos, revoca y anula todos los testamentos, codicilos y otras voluntades realizadas antaño y redacta su último testamento ${ }^{9}$; del que destacan por su trascendencia dos cláusulas (la referida a su enterramiento en la cartuja castellonense de Valldecrist - como ya lo hizo su hermanoy la que habla del legado de la mencionada alquería, con todas sus tierras, un molino harinero y todos los bienes inmuebles allí hallados, al cenobio antedicho).

\footnotetext{
${ }^{3}$ Benito, F. La arquitectura del Colegio del Patriarca y sus artífices, Valencia, 1981, pp. 47-48 y «Real Colegio del Corpus Christi o del Patriarca», Catálogo de monumentos y conjuntos de la Comunidad Valenciana, tomo II, Conselleria de Cultura, Educació i Ciència de la Generalitat Valenciana (ed . bilingüe), Valencia, 1983, p. 405; Bustamante, A.-Marías, F. art. cit., pp. 56-57; Aranda, F.- Fernández, M. «La cúpula del Real Colegio del Corpus Christi de Valencia», $A r$ quitectura Técnica, 4, diciembre 1988, pp. 36-42; Benito, F. «El Reial Col.legi Seminari de Corpus Christi», La Universitat de València i el seu entorn urbà, Universitat de València, València, 2001, pp. 306-309; Benito, F.-Bérchez, J. «Iglesia y convento del Carmen», Catálogo de monumentos ..., tomo II, Valencia, 1983, pp. 442 y 444 o Arciniega García, L. El monasterio de San Miguel de los Reyes, Biblioteca Valenciana, vol. I, Valencia, 2001, pp. 177-178.

${ }^{4}$ Sobre el particular han polemizado F. Pingarrón, que la cree realizada antes de 1631 («A propósito de la arquitectura de la primitiva iglesia de la Compañía de la ciudad de Valencia», Archivo de Arte Valenciano, LXVII, 1986, pp. 27-34), y M. Gómez-Ferrer, que intenta demostrar que fue finalizada en 1631 («La iglesia de la Compañía de la ciudad de Valencia. El contrato para la finalización de las obras de su cabecera en 1621», Archivo de Arte Valenciano, LXXIV, 1993, pp. 5658). Tal vez, proceda resaltar que Francesc Arboreda, maestro de obras encargado de la conclusión de la cabecera (y cúpula) a partir del 28 de octubre de 1621, formó parte de la comisión encargada de comprobar la buena arquitectura del cenobio (con su iglesia y cúpula) del Puig el 18 de septiembre de 1621 . Asimismo, nos remitimos a las deliberaciones sobre las trazas del complejo monacal, donde se percibe cierto paralelismo entre ambas fábricas en lo concerniente a sus estructuras extradosadas.

${ }^{5}$ Serra Desfilis, A. Gandia, 450 anys de tradició universitària (1549-1999), 1999, pp. 68-69.

${ }^{6}$ Las fundaciones cartujanas en el reino de Valencia fueron: Porta Coeli (1272), Valldecrist (1385), La Anunciación de María Santísima (1442), Ara Christi (1585) y Via Coeli (1640).

${ }^{7}$ La heredad había pertenecido a mosén Cristòfor Roig, hermano de doña Elena e inquisidor apostólico en Zaragoza y chantre de la Seo valenciana, quien debió adjudicarla en su testamento (recordemos que falleció el 18 de febrero de 1581) a la fundadora de Ara Christi.

${ }^{8}$ Archivo Histórico Nacional (= A.H.N.), Clero, carpeta 3.146, pergamino $\mathrm{n}^{\circ} 16$.

${ }^{9}$ Archivo de Protocolos del Patriarca de Valencia (= A.P.P.V.), protocolos de Jaume Cristòfor Ferrer, $\mathrm{n}^{\circ} 10.095$.
}

AEA, LXXVIII, 2005, 309, pp. 71 a 81 


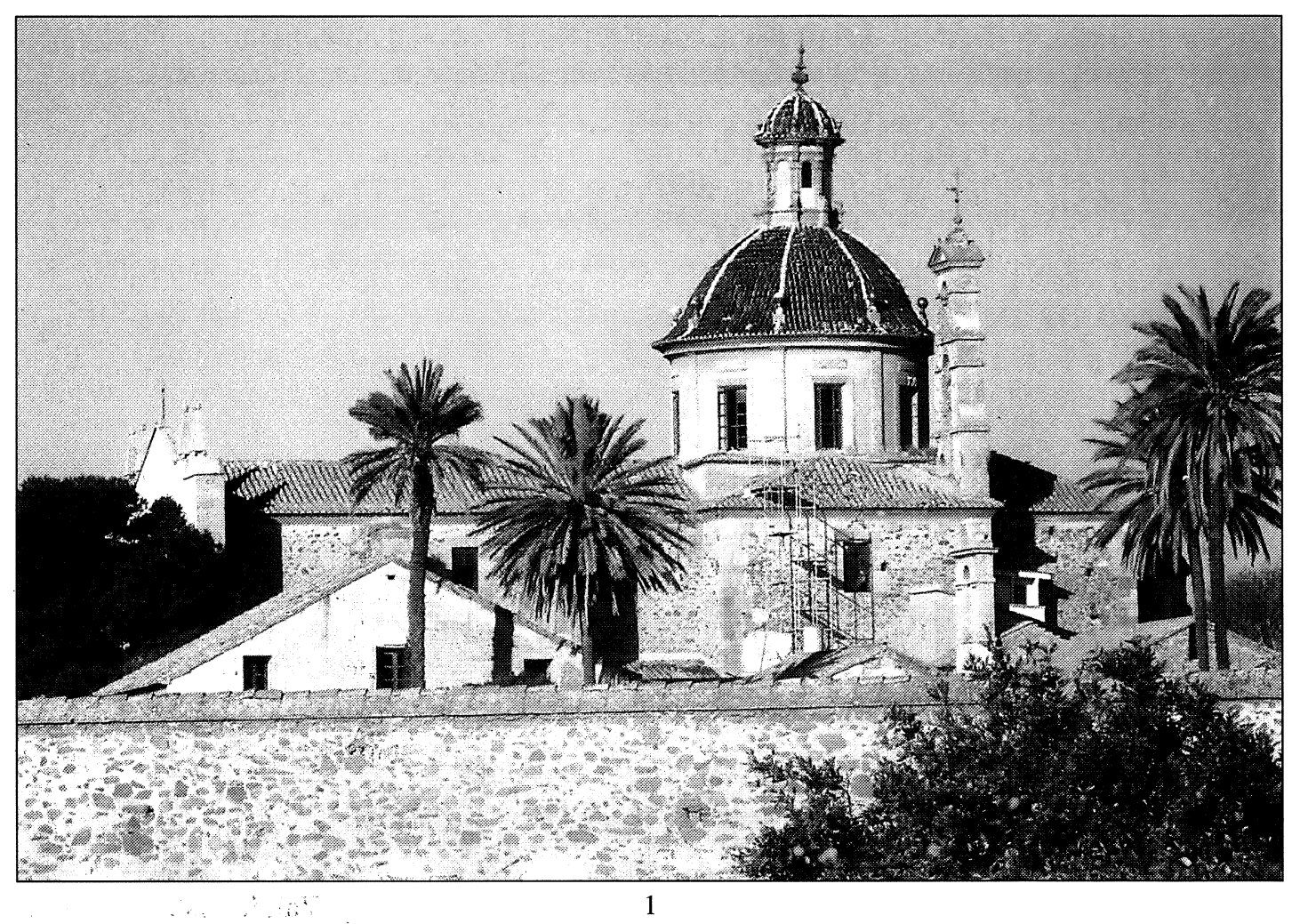

2
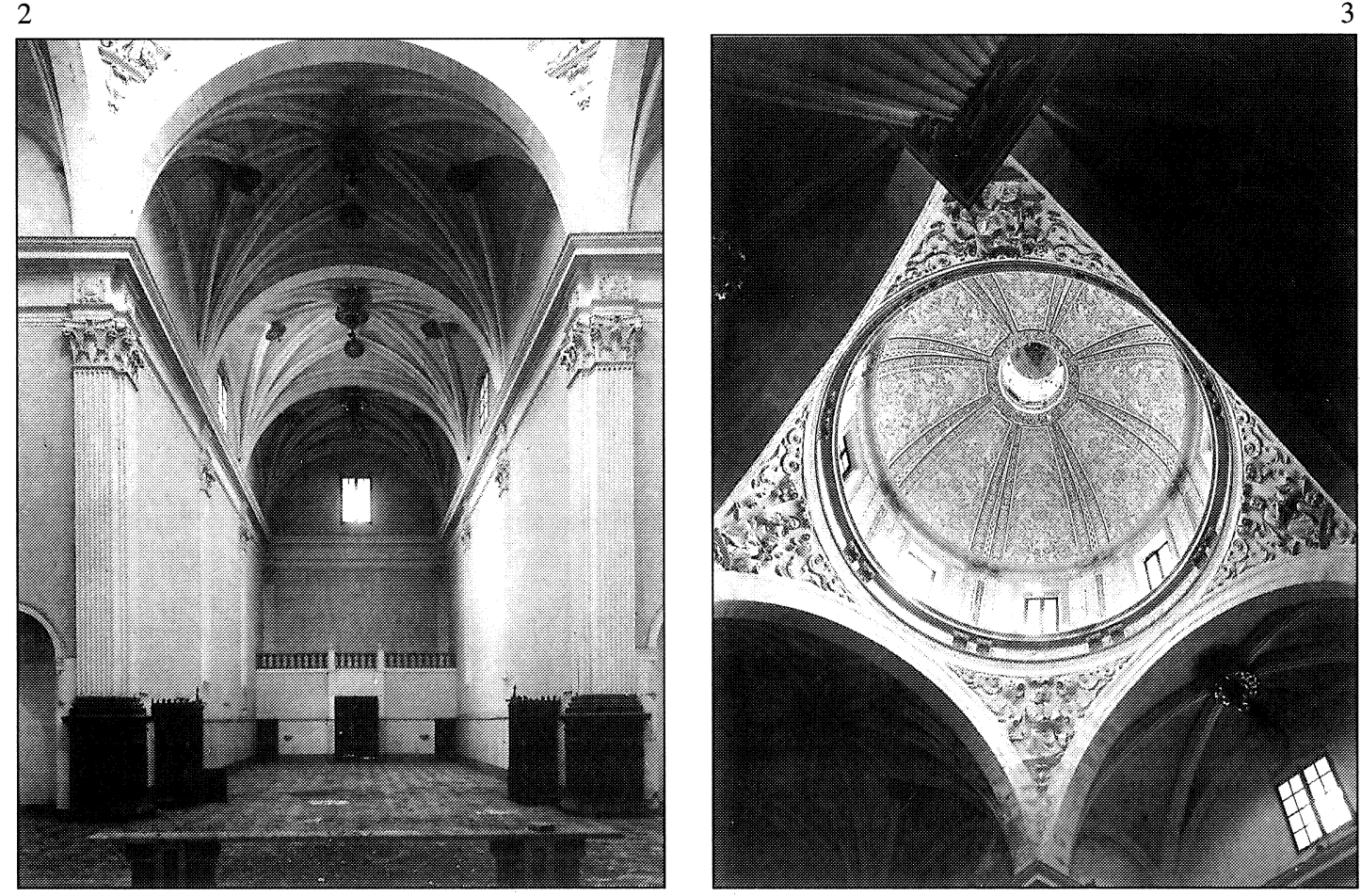

Fig. 1. Iglesia desde levante.

Fig. 2. La nave del templo desde el altar (F. Zubillaga).

Fig. 3. Intradós de la cúpula (F. Zubillaga).

$A E A$, LXXVIII, 2005, 309, pp. 71 a 81 
No obstante, estas voluntades sufrieron un súbito cambio el 19 de agosto de 1584, según se desprende del codicilo que al testamento de 1582 realizó doña Elena, pues revoca ambas cláusulas y redacta otras nuevas: «És ma voluntat que sobre dit llegat de la Alqueria, que direm per nom Nostra Señora de Arachristi, sia fundada casa de Cartuxos per a que allí sia fundat Monestir que se alabe lo nom de Nostre Señor Déu per a tots los temps. Més és ma voluntat que los pares que la fundaran dita casa de Nostra Señora de Arachristi no sien subiectes a ningun convent de dita cartoxa [ ... ]. Més és ma voluntat que si al temps de la mia mort esta casa no estara en lo aparell ques pogués fer, fos portat lo meu cos y acomanat en Sant Agostí, en la mia capella de Sant Pere, fins que en el dit monestir de Nostra Señora de Arachristi se puga portar, lo qual supplique molt a mos marmesors que estan en dit testament, rebut per lo sobredit notari, es donen tota la presa posible» ${ }^{10}$.

Todo indica que cuando doña Elena Roig decidió testar sus parientes directos habían fallecido, por lo que es posible que ésta acumulara las heredades de los finados y quizás suponiendo que su muerte estaba próxima, pensó repartir su considerable herencia al resto de su familia, al personal de servicio, religiosos, parroquias, conventos, ... teniendo muy en cuenta - probablemente - la opinión o sugerencias de su cualificado círculo de amistades (el ermitaño Pere Muñoz, el franciscano fray Jaume Sanchis, el jesuita Martí Alberro o Isabel Joan Amigó, ...) en lo concerniente al codicilo ${ }^{11}$.

Producido el óbito de la fundadora el 1 de abril de 1585 , creemos que sus últimas voluntades eran conocidas con seguridad por los monjes; quienes se encargaron, antes de la defunción de la testadora, de solicitar a las autoridades competentes las licencias necesarias para poder fundar un nuevo cenobio cartujo ${ }^{12}$. Unos días más tarde tomaron posesión de la hacienda fray Sanxo Anyó y fray Joaquim Amigó, como profesos de Porta Coeli y Valldecrist, respectivamente, y fray Miquel de Vera como primer prior de la fundación, ante el notario de Valencia Joan Miquel ${ }^{13}$.

Una vez admitida la nueva casa en la Orden por su capítulo general de $1585^{14}$, los problemas surgieron pronto. Así, entre la confusión de los primeros momentos, fue nombrado como prior el padre Amigó, en sustitución de Vera, quien había marchado a Porta Coeli ${ }^{15}$. Durante su mandato viajó a la Corte y, al tiempo que consiguió la licencia necesaria por el patriarca Ribera el 29 de abril de $1586^{16}$, alentó la construcción de la iglesia primitiva, que posteriormente pasó a formar parte de la conrería, obra de Joan Casaña ${ }^{17}$. A pesar del esfuerzo por afirmar y normalizar la situación del cenobio, continuado por los sucesivos priores (fray Pere Samaniego, fray Josep Ferrer, otras dos veces por el padre Amigó, fray Miquel Monllor y fray Miquel Diça) surgió la posibilidad de clausurarlo ${ }^{18}$. Acto que nunca se llevó a efecto porque con el nombramiento de fray Pere Mateo, profeso de Porta Coeli, como nuevo prior se paralizó la disgregación.

Este nuevo prior, gracias a su amistad con Andreu Capilla —obispo de Urgell y antiguo

\footnotetext{
${ }^{10}$ A.H.N., Clero, carpeta 3.146 , pergamino $\mathrm{n}^{\circ} 16$.

${ }^{11}$ Ferrer Orts, A. La reial cartoixa de Nostra Senyora d'Ara Christi, Ajuntament del Puig, 1999, p. 56.

${ }_{12}$ A.H.N., Clero, carpeta 3.146, pergamino $\mathrm{n}^{\circ} 17$.

${ }^{13}$ A.H.N., Clero, carpeta 3.146, pergamino $\mathrm{n}^{\circ} 18$ y A.P.P.V., protocolos de Joan Miquel, $\mathrm{n}^{\circ} 2.115$.

${ }^{14}$ Archivo del Reino de Valencia (= A.R.V.), Clero, libro ${ }^{\circ}$ 2.959, s.f.

${ }^{15}$ Las vicisitudes de estos primeros y cruciales momentos de la fundación de l'Horta quedaron recogidos por este primer rector en el manuscrito conservado posiblemente en el Archivo de la Grande Chartreuse con el título Domus ca(rtusia)e Arae-Christi, in Regno Valentino, provinciae Cathaloniae ... suae fundationis, anno d(omi)ni 1584, principium in hunc modum habuit. A pesar de no haber podido consultar el texto por encontrarse en proceso de catalogación los fondos del mencionado archivo, agradecemos a Dom Luc Fauchon su interés por satisfacer nuestras peticiones.

${ }^{16}$ Ferrer Orts, A. op. cit., p. 205.

17 Ferrer Orts, A. op. cit., p. 87.

${ }^{18}$ Ferrer Orts, A. op. cit., p. 58.
} 
monje cartujo ${ }^{19}$ - impulsó la edificación de algunas obras de efímera existencia ${ }^{20}$, toda vez que heredara del prelado una cuantiosa suma además de otras propiedades ${ }^{21}$, con la finalidad de incrementar el patrimonio de los conventuales como de mantener un número mayor de éstos. A pesar de ello, su rectorado conoció otros hechos relevantes, como la complicada adquisición de las tierras y alquería colindantes a los hermanos Lledó en junio de 1608 (compra que, a la postre, resultará vital para el desarrollo futuro del complejo monacal) ${ }^{22} \mathrm{y}$, por otra, el enfrentamiento desatado con los habitantes del Puig por el desvío del camino real en marzo de 1611 (indispensable para acceder a una mayor intimidad y recogimiento espiritual y, de paso, dar sentido a ambas propiedades) ${ }^{23}$.

Los sucesores inmediatos del padre Mateo, fray Francesc Font y fray Bertomeu Puig, además de continuar la obra del claustro de la conrería - en la que intervinieron fray Antoni Ortiz, Tomàs Panes, Pere Della, Francesc Sayas y Joan Sales ${ }^{24}$-, de la portería — debida a Pere Conde y al citado Ortiz ${ }^{25}$ - y de los muros del recinto - por Andreu Pons, Pere Navarro, Vicent Valentí, Joan Vila i Joan Lluch ${ }^{26}$-, estudiaron con la comunidad franciscana de Santo Espíritu del Monte de Gilet la posibilidad de intercambiar ambos cenobios. Actuaciones que quedaron en papel mojado cuando llegaron a oidos del capítulo provincial franciscano ${ }^{27}$.

Pasados treinta años desde la toma de posesión de las heredades de Elena Roig por parte cartujana, el cenobio apenas había evolucionado. Si bien es cierto que sus rentas se acrecentaban paulatinamente, no lo es menos que su plasmación arquitectónica, lejos de ser uniforme, parece que no obedecía a ningún plan director ideado antaño. Habrá que esperar al priorato de fray Francesc Almenar (1615-1624) ${ }^{28}$, profeso de Aula Dei, para vislumbrar lo que será la verdadera planificación de la cartuja y su impulso edilicio posterior.

Como decimoprimer prior de Ara Christi, el padre Almenar - arropado de las suficientes rentas para emprender con éxito la edificación de un monasterio de nueva planta- puso en práctica lo que a buen seguro rondaba en su cabeza desde su llegada al Puig. Paso previo a ello podemos considerar la realización de un horno por fray Pere Ruimonte y fray Martí de Cinto desde marzo de 1619, así como una bodega a cargo de Pere Panes desde septiembre de $1620^{29}$; fechas que coinciden plenamente con las deliberaciones previas a su traza definitiva y futura construcción.

Según relata detalladamente fray Honorat Navarro al describir las vicisitudes de la fundación ${ }^{30}$, en el año 1619 se iniciaron los preparativos con la discusión previa sobre las trazas del complejo monástico por parte de los maestros de obras Valer Planes ${ }^{31}$ y del cartujo fray Pere

\footnotetext{
${ }^{19}$ Capilla (también conocido como Capella) profesó por dos veces como cartujo, fue prior de Porta Coeli, El Paular y Scala Dei de donde pasó a regentar por voluntad regia el obispado de Urgell desde 1587. Sobre su figura se han ocupado, entre otros, Vallés, Tarín y Juaneda, Gort, Moliné, Ribes Traver o el que aquí suscribe.

${ }^{20}$ Como la capilla que empezó a fabricar (y parece que nunca se concluyó) dedicada a San Antonio Abad el 25 de enero de 1602. Recordemos que el obispo Capilla también se erigió un ermitorio en Montsant, cerca de Scala Dei.

${ }^{21}$ Ferrer Orts, A. op. cit., pp. 58 y 69.

${ }^{22}$ A.P.P.V., protocolos de Vicent Onyate, $\mathrm{n}^{\circ} 16.946$

${ }^{23}$ Ferrer Orts, A. op. cit., pp. 58-59.

${ }^{24}$ Ferrer Orts, A. op. cit., pp. 82, 86 y 87.

25 Ibid.

26 Ibid.

${ }^{27}$ Estas conversaciones ya se habían iniciado en tiempos del primer priorato de fray Joaquim Amigó, quedando interrumpidas hasta el rectorado de fray Bertomeu Puig.

${ }^{28}$ De Almenar se han ocupado Bosqued, Barlés Báguena o el autor de estas líneas.

${ }^{29}$ Ferrer Orts, A. op. cit., pp. 82 y 87.

30. Este documento, al que haremos referencia a partir de ahora, se encuentra íntegramente transcrito en el apéndice documental de nuestra comunicación «En torno a la actividad constructiva en el reino de Valencia durante la primera mitad del siglo XVII. La cartuja de Ara Christi (El Puig)», Actes del XVII Congrés d'Història de la Corona d'Aragó (separata), Barcelona-Lleida, septiembre, 2000, pp.12-14.

${ }_{31}$ Mencionado como «arquitecte de la iglesia de les monjes de Sogorb «(se refiere al templo del monasterio de San Martín, erigido entre 1600-1622 según M.Gómez-Ferrer). Véase al respecto Ferrer Orts, A. «En torno a la actividad ...», Actes del XVII Congrés d'Història de la Corona ..., p. 4.
} 


\begin{tabular}{|l|l|c|c|}
\hline \multicolumn{5}{|c|}{ CARTUJA DE ARA CHRISTI } \\
\hline \multicolumn{2}{|c|}{ TRAZAS } & \multicolumn{2}{c|}{ PERÍODO (1619-1621 ) } \\
\hline \multicolumn{1}{|c|}{ ARTÍFICE } & \multicolumn{1}{|c|}{ PROFESIÓN } & CRONOLOGÍA & ACTUACIONES \\
\hline FRANCESC CATALÀ & Maestro de obras & $1620-1621$ & \\
\hline FRA ANTONI ORTIZ & Maestro de obras & $1619-1621$ & \\
\hline TOMÀS PANES & Maestro de obras & $1619-1621$ & \\
\hline VALER PLANES & Maestro de obras & $1619-1621(?)$ & \\
\hline MOSSÉN GUILLEM ROCA & Maestro de obras & $1620-1621$ & \\
\hline FRA PERE RUIMONTE & Maestro de obras & $1619-1621$ & \\
\hline FRA G. DE SANMARTÍ & Arquitecto & $1619-1621$ & \\
\hline JOAN BTA. VERGARA & Carpintero & 1621 & \\
\hline
\end{tabular}

Tabla 1.

Ruimonte ${ }^{32}$, tomando como ejemplo a seguir la planta de la cartuja de Aula Dei ${ }^{33}$. Fracasado este primer intento, se reemprendieron de nuevo las conversaciones en enero de 1620, fecha en la que fueron citados por el padre Almenar el arquitecto carmelita fray Gaspar de Sanmartí y los maestros de obras Francesc Català, mosén Guillem Roca, Ruimonte y fray Antoni Ortiz, así como el padre fray Francesc Font, prior de Porta Coeli (y antaño de Ara Christi), y después de las objeciones de los presentes a los diseños de Ruimonte, así como de la clara voluntad del padre Almenar de ajustarse en lo posible a la traza del conjunto aragonés porque «[ ... ] es la mejor que hay en España en edifiçio y disposiçión de offiçinas [ ... ]» ${ }^{34}$, quedó reprobada la traza del cartujo y encomendada su ejecución a Sanmartí (con la colaboración de Roca en lo referido a la disposición de la iglesia) ${ }^{35}$.

Aclarada, por tanto, la autoría de los planos, comenzaron los preparativos para la construcción de la nueva cartuja, que recibió el espaldarazo definitivo cuando en febrero de 1621 fueron de nuevo convocados los expertos arriba mencionados - excepto Roca-, a los que se incorporó el maestro de obras Tomàs Panes, así como fray Andrés Álvaro como nuevo prior de Porta Coeli (y posteriormente de Ara Christi). Pues en esas fechas se señalaron sobre el terreno las dimensiones de la iglesia y del claustro mayor, a la vez que se redactaron una serie de capitulaciones muy escuetas y concisas ${ }^{36}$, tras lo que se procedió a la colocación de la primera piedra de la iglesia ${ }^{37}$.

Superado este primer estadio, los problemas volvieron a sacudir nuevamente el sosiego cartujano, ya que en septiembre de 1621 otra comisión se personó en el Puig con la intención de informarse si la traza, los cimientos y los materiales empleados, así como el lugar elegido reunían las mínimas condiciones de buena arquitectura y salubridad. Después de muchos sufrimientos por parte del prior y de sus conventuales, los informes de los expertos requeridos resultaron satisfactorios y el 18 de noviembre de este año el General de la Orden permitió la reanudación de las obras ${ }^{38}$.

Vuelta la normalidad a la fundación, ya no se detuvo el progreso de su fábrica, especialmente intenso en los próximos sesenta años. Muestra de ello es la ejecución de gran parte del cenobio, como la iglesia (1621-1640), el claustro mayor (1625-1673), el trasagrario (16301639), la sacristía (1631-1633), las celdas (1630-1673), las capillas de Nuestra Señora del Pilar y de San José (1631-1639), la celda del sacristán (1632-1639), la celda prioral (1630-1633),

\footnotetext{
${ }^{32}$ Barlés Báguena, E. «La participación de los miembros de las órdenes religiosas en el proceso de construcción de sus monasterios. El caso de la orden cartujana en España (siglos XVII y XviII)», Artigrama, 10, 1993, pp. 329, 330 y 343. Así como Ferrer Orts, A. «En torno a la actividad ...», Actes del XVII Congrés d'Història de la Corona ..., pp. 4 y 8.

${ }^{33}$ Barlés Báguena, E. art. cit., pp. 328-331 y Ferrer Orts, A. «En torno a la actividad ...», Actes del XVII Congrés d'Història de la Corona ..., pp. 4 y ss.

${ }^{34}$ Ferrer Orts, A. «En torno a la actividad ...», Actes del XVII Congrés d'Història de la Corona ..., pp. 4 y 12

${ }^{35}$ Ferrer Orts, A. «En torno a la actividad ...», Actes del XVII Congrés d'Història de la Corona ..., pp. 12-13 .

${ }^{36}$ Ferrer Orts, A. «En torno a la actividad ...», Actes del XVII Congrés d'Història de la Corona ..., pp. 12-14.

37 A.R.V., Clero, libro n ${ }^{\circ}$ 2.312, s.f. y A.H.N., Códices, 1.372 B., fs. 74 r. -75 v.

38 A.H.N., Códices, 1.372 B, fs. 76 v. -90 r.
}

AEA, LXXVIII, 2005, 309, pp. 71 a 81 
el claustro de levante (1635-1639), el claustro de poniente (1650-160), el aula capitular (16501657) o el refectorio (1656-1678) ${ }^{39}$, donde participaron gran cantidad de artífices ${ }^{40}$.

\section{LA IGLESIA (1621-1640)}

Entre 1619 y el 6 de febrero de 1621, período en el que transcurren las deliberaciones sobre la traza de la cartuja, la planificación de la iglesia (y de su cúpula) ocupa la mayor parte de los esfuerzos de los expertos convocados a tal efecto. Así lo refleja el valioso testimonio de fray Honorat Navarro : "[ ... ] En lo ayn següent de 1620, a 17 de Jiner, tinguerem Junta de Mestres per a traçar la Iglesia y casa,y axi foren cridats fr(a) Gaspar de Semartí, religiós del Carme, mestre Frances Cathalà y mossén Guillem Roca, fr (a) Pedro Ruimonte y fr(a) Anthoni Orti [...] y tot lo matí trastejaren la traça y, aprés de dinar, començaren a traure lo compàs y a traçar [ ... ]. Vist asò, posàs lo Cathalà a traçar la Iglesia y sobre repartir les navades trobaven molta difficultat, prengué lo compàs mossén Roca y també no se'n desexia, fins a que fr(a) Gaspar y ell se encarregaren de juntar-se en la Eliana. Però fr(a) Gaspar parlà al pare Prior de secret que ell se encarregava no obstant que, sense llevar la honra a algú, Roca sap més que fr(a) Gaspar y Cathalà, perquè ell des de València, per medi de terçeres persones nos avisara o els [... ] conforme fon en la cruçeria de la Iglesia que havien traçat un cruzeros de un palm de llargària, que si Roca nou advertira al pare Alfonso de la Compañia per a que sus avisàs, feem la maior boveria del món. També en lo redó del çimbori que havia de dexar Mellado per a que ell lo edificara de alli amunt, també si no fora per Roca forem perduts perquè ni se hauria dexat punt redó ab los tirantes de les pechines de ninguna manera y ell ho remedia ab molta façilitat, y després ha corregit mil faltes y ha dispost la traça de manera que serà una de les perfectes obres que haurà plaent al Senyor $[\ldots]{ }^{41}$.

Una vez puestos de acuerdo «[...] después de muchas disputas y altercados, con pareçer y unánime consentimiento de todos, se resolvieron las cosas siguientes para que los presentes y venideros sepan la forma y modo que han de guardar en la execuçión de la dicha fábrica y casa nueva que hoy, día de la gloriosa sancta Dorothea que contamos a 6 de Febrero deste presente año 1621, se han principiado a abrir los fundamentos en el lugar donde ha de estar el altar maior de dicha iglesia [...]» ${ }^{42}$, se redactaron un total de ocho cláusulas a modo de escuetas capitulaciones con la conformidad de Sanmartí, Català, Panes, Ortiz, Ruimonte, así como de los priores de Porta Coeli y Ara Christi y del propio padre Navarro en calidad de vicario ${ }^{43}$. El 4 de marzo de 1621 se procedió a colocar la primera piedra del templo con gran pompa ${ }^{44}$, honor que recayó en fray Antoni Ortiz quien, a su vez, había delegado en el mampostero Bartomeu Fontanilla la tarea de levantar el templo ${ }^{45}$ (actuación que se prolongó durante casi un lustro y que contó con la destacada colaboración entre 1621-1622 de su oficial Joan Navarro y de un cantero milanés llamado Domènec) ${ }^{46}$.

\footnotetext{
${ }^{39}$ Ferrer Orts, A. «En torno a la actividad ...», Actes del XVII Congrés d'Història de la Corona ..., pp. 1-16.

${ }^{40}$ Ferrer Orts, A. «La historiografia de la cartoixa valenciana d'Ara Christi i els seus artífexs (1585-1660) », Boletín de la Sociedad Castellonense de Cultura, LXXVIII, 2002, pp. 415-459.

${ }^{41}$ Ferrer Orts, A. «En torno a la actividad...», Actes del XVII Congrés d'Història de la Corona ..., pp. 12-13.

${ }^{42}$ Ferrer Orts, A. «En torno a la actividad ...», Actes del XVII Congrés d'Història de la Corona ..., p. 13.

${ }^{43}$ Ferrer Orts, A. «En torno a la actividad ...» Actes del XVII Congrés d'Història de la Corona ..., pp. 13-14.

${ }^{44}$ Véase la nota 37.

${ }^{45}$ Ferrer Orts, A. op. cit., pp. 83 y 88, y del mismo autor «En torno a la actividad ...», Actes del XVII Congrés d'Història de la Corona ..., pp. 6-7, y «La historiografia de la cartoixa ...», Boletín de la Sociedad Castellonense de Cultura, LXXVIII, 2002, pp. 431 y 444.

${ }^{46}$ Ferrer Orts, A. «La historiografia de la cartoixa ...», Boletín de la Sociedad Castellonense de Cultura, LXXVIII, 2002, pp. 427 y 440 .
}

$A E A$, LXXVIII, 2005, 309, pp. 71 a 81 


\begin{tabular}{|c|c|c|c|}
\hline \multicolumn{4}{|c|}{ CARTUJA DE ARA CHRISTI } \\
\hline \multicolumn{2}{|c|}{ IGLESIA } & \multicolumn{2}{|c|}{ PERÍODO ( 1621-1640) } \\
\hline ARTÍFICE & PROFESIÓN & CRONOLOGÍA & ACTUACIONES \\
\hline ANDREU ARTIC & Oficial & 1627 & $\begin{array}{l}\text { Encerado del templo y } \\
\text { capiteles de las pilastras. }\end{array}$ \\
\hline ANTONI BADENES & Maestro de obras & $1631-1634$ & $\begin{array}{l}\text { Linterna y calota de la cú } \\
\text { pula, tejado y portada del } \\
\text { templo. }\end{array}$ \\
\hline A. BADENES ( hno. de ) & Peón (?) & $1631-1632$ & Portada del templo. \\
\hline MARC BONOS & Maestro de obras & 1631 & $\begin{array}{l}\text { Alabastro del templo y la } \\
\text { cúpula. }\end{array}$ \\
\hline FRANCESC CATALÅ & Maestro de obras & 1624 & Cubrición del templo. \\
\hline JOAN CLARAMUNT & Maestro de obras & 1674 & Linterna y cúpula. \\
\hline DOMÈNEC, milanés & Cantero & $1621-1622$ & $\begin{array}{l}\text { Cimientos y mazonería del } \\
\text { templo. }\end{array}$ \\
\hline P. FOIX & Maestro de obras & 1631 & $\begin{array}{l}\text { Alabastros del templo y } \\
\text { cimborrio. }\end{array}$ \\
\hline BARTOMEU FONTANILLA & Maestro de obras & $1621-1625$ & Mazonería del tempio. \\
\hline URBÀ FOS & Pintor & $1651(?)$ & Retablo mayor. \\
\hline ESTEVE GRAMALLES & Maestro de obras & 1621 y 1654 & \\
\hline R. HERRERO DEL REAL & Herrero & 1631 & Templo y cúpula. \\
\hline RAFAEL MALLORQUÍ & Herrero & $1632-1639$ & Cúpula y coro. \\
\hline MIQUEL MARTÍNEZ & Librero & 1640 & Templo (?) \\
\hline MATEU & Librero & 1640 & Coro. \\
\hline TOMÅS MELLADO & Cantero & $1621-1636$ & $\begin{array}{l}\text { Pedestales y pechinas de la } \\
\text { cúpula, arcadas, claves y } \\
\text { gradas del templo. }\end{array}$ \\
\hline JOAN NAVARRO & Oficial & $1621-1622$ & Templo. \\
\hline JOAN MIQUEL ORLIENS & Escultor & $1625-1626(?)$ & Claves de la bóveda. \\
\hline FRA ANTONI ORTIZ & Maestro de obras & 1621 y $1626-1627$ & Alabastro del templo. \\
\hline MIQUEL OVIEDO & Escultor & 1627 & $\begin{array}{l}\text { Capiteles del templo y } \\
\text { evangelistas de las pe- } \\
\text { chinas de la cúpula. }\end{array}$ \\
\hline FRANCESC PADILLA & Maestro de obras & 1674 & Linterna y cúpula. \\
\hline TOMÅS PANES & Maestro de obras & $1621-1627$ & $\begin{array}{l}\text { Cubrición del templo y cú } \\
\text { pula. }\end{array}$ \\
\hline PAU & Oficial & $1631-1637$ & Linterna de la cúpula. \\
\hline JAUME REBULL & Maestro de obras & 1643-1647 & Cúpula. \\
\hline DOMENNEC REDOLAT & Maestro de obras & $1636-1650$ & Fachada (?) \\
\hline MOSSÉN GUILLEM ROCA & Maestro de obras & 1621 y $1625-31$ & $\begin{array}{l}\text { Cúpula, alicatado del } \\
\text { templo y capillas laterales. }\end{array}$ \\
\hline JORDI SIBILE & Pintor & $1651-1653(?)$ & Retablo mayor (?) \\
\hline JOAN BTA. TORMO & Escultor & $1651-1653$ & Retablo mayor. \\
\hline ANTONI TORRERA & Maestro de obras & 1635 & Cúpula. \\
\hline VICENT VALENTÍ & Calero & 1621 & Cimientos del templo. \\
\hline JOAN BTA. VERGARA & Carpintero & $1621-1660(?)$ & $\begin{array}{l}\text { Grúas, cúpula y puertas del } \\
\text { templo. }\end{array}$ \\
\hline JOAN VIDAL & Campanero & 1640 & Espadaña y templo. \\
\hline
\end{tabular}

Tabla 2.

Al mismo tiempo, el cantero de Valencia Tomàs Mellado, activo en el cenobio entre 16211636, aparece cobrando 200 libras por los cuatro pedestales del cimborrio de la iglesia en agosto de 1621, así como realizando portes de piedra de Godella, Riba-roja y Porta Coeli, acabando las arcadas y las pechinas del crucero, ocupado en las claves pétreas para las bóvedas nervadas y ejecutando la portada y las gradas de la misma; actuaciones que debieron finalizar en $1624^{47}$. Año en el que los maestros de obras Francesc Català y Tomàs Panes concertaron la cubrición de la iglesia, labor que prosiguió el segundo desde noviembre de 1624 por el óbito del primero ${ }^{48}$.

${ }^{47}$ Barlés Báguena, E. art. cit., p. 341 y Ferrer Orts, A. op. cit., pp. 83, 84, 88 y 89, «En torno a la actividad ...», Actes del XVII Congrés d'Història de la Corona ..., p. 6, y «La historiografia de la cartoixa ...», Boletín de la Sociedad Castellonense de Cultura, LXXVIII, 2002, p. 438-439.

${ }^{48}$ Barlés Báguena, E. art. cit., pp. 330, 341, 343 y 344; Ferrer Orts, A. op. cit., pp. 83 y 88, «En torno a la actividad ...», Actes del XVII Congrés d'Història de la Corona ..., pp. 5 y $7, \mathrm{y}$ «La historiografia de la cartoixa ...», Boletín de la Sociedad Castellonense de Cultura, LXXVIII, 2002, pp 423 y 446.

$A E A$, LXXVIII, 2005, 309, pp. 71 a 81 
En 1625, y muy probablemente hasta julio de 1626, encontramos al escultor oscense Joan Miquel Orliens dorando y pintando las claves de madera (a modo de grandes florones) que para la iglesia había realizado ${ }^{49}$. A partir de noviembre de 1625 reaparece el maestro de obras mosén Guillem Roca, quien permanecerá ligado a diversas actuaciones en el complejo cartujano hasta julio de 1631, año de su fallecimiento. Sin embargo, lo que nos interesa es resaltar su participación en el alicatado de la iglesia, en la sacristía, en las capillas laterales y, particularmente, en la elevación de la cúpula del templo, concertada ante el notario de Valencia Bru Muñoz por 1.920 libras ${ }^{50}$.

De 1627 data la colaboración de Andreu Artic y de Miquel de Oviedo, oficial e «imaginari» de Roca respectivamente, en los capiteles de las pilastras del templo ${ }^{51}$. Individualmente habían laborado en el encerado del edificio el primero y, posiblemente, en dar forma a los cuatro evangelistas el segundo ${ }^{52}$.

Más adelante, entre junio de 1631 y diciembre de 1634, se documenta la actividad del maestro de obras Antoni Badenes en la cartuja (principalmente en la cúpula y su linterna, en el tejado, en la puerta principal y en la sacristía de la iglesia) ${ }^{53}$. Así como del herrero Rafael Herrero del Real rematando los ocho ventanales del tambor de la cúpula - previamente realizadas por el carpintero Joan Baptista Vergara - y otras cinco para el resto del templo, además de la cruz de hierro que remataba la calota (1631); y de los maestros de obras Marc Bonos y P. Foix en el destajo de los alabastros de las ventanas de la iglesia y del tambor de la cúpula, previamente traídos de Aragón por fray A. Ortiz, entre julio y octubre de 1631, el primero, y en noviembre del mismo, los dos juntos ${ }^{54}$. Del mismo modo, se tiene noticia de la actuación de un oficial de Martí d'Orinda llamado Pau en el intervalo que va desde el mes de agosto de 1631 hasta mayo de 1637, especialmente en la linterna de la cúpula ${ }^{55}$, juntamente con el maestro de obras Antoni Torrera ${ }^{56}$, por causa de los daños ocasionados por un rayo.

Entre los meses de agosto de 1632 y diciembre de 1639 el herrero Rafael Mallorquí se encargó de materializar la cruz que remataba la cúpula del trasagrario y dos anillos para la misma, los preparativos para la sillería del coro, así como las rejas y las cerraduras de las puertas del mencionado reservado ${ }^{57}$. Mientras que el maestro de obras Domènec Redolat, relacio-

\footnotetext{
49 Barlés Báguena, E. art. cit., p. 341 y Ferrer Orts, A. op. cit., pp. 83, 88 y 89, «En torno a la actividad ...», Actes del XVII Congrés d'Història de la Corona ..., p. 7, y «La historiografia de la cartoixa ...», Boletín de la Sociedad Castellonense de Cultura, LXXVIII, 2002, p. 442-443.

${ }^{50}$ Barlés Báguena, E. art. cit., pp. 330 y 341; Ferrer Orts, A. op. cit., pp. 83 y 89, «En torno a la actividad ...», Actes del XVII Congrés d'Història de la Corona ..., p. 8, y «La historiografia de la cartoixa ...», Boletín de la Sociedad Castellonense de Cultura, LXXVIII, 2002, p. 450-451.

${ }^{51}$ Ferrer Orts, A. op. cit., pp. 83-89, «En torno a la actividad ...», Actes del XVII Congrés d'Història de la Corona ..., p. 7, y «La historiografia de la cartoixa ...», Boletín de la Sociedad Castellonense de Cultura, LXXVIII, 2002 , pp. 417 y 444. 52 Ibid.

53 Barlés Báguena, E. art. cit., p. 341 y Ferrer Orts, A. op. cit., pp. 84 y 89, «En torno a la actividad ...», Actes del XVII Congrés d'Història de la Corona ..., p. 5, y «La historiografia de la cartoixa ...», Boletín de la Sociedad Castellonense de Cultura, LXXVIII, 2002, p. 417-418.

${ }^{54}$ Ferrer Orts, A. «La historiografia de la cartoixa ...», Boletín de la Sociedad Castellonense de Cultura, LXXVIII, 2002, pp. 420, 430, 434 y 458 . Barlés Báguena, E. «Historia constructiva de la cartuja de Ara Christi (El Puig, Valencia) 15851835», Artigrama, 17, 2002, pp. 369-370 propone como fecha de finalización del cimborrio (y de la cúpula que lo remataba) 1629 , dos años antes de su definitiva culminación estructural según nuestras investigaciones. Igualmente discrepamos de la cronología que dicha autora considera para la ejecución de la decoración esgrafiada del intradós de la media naranja (1627), pues no sólo estaba construyéndose la calota por ese período, sino que además creemos documentar su exornación a partir de 1642 .

${ }^{55}$ Ferrer Orts, A. «La historiografia de la cartoixa ...», Boletín de la Sociedad Castellonense de Cultura, LXXVIII, 2002, p. 446. Sobre la participación de Orinda, véase Barlés Báguena, E. art. cit., pp. 339 y 341 y Ferrer Orts, A. op. cit., pp. 84 y 89, «En torno a la actividad ...», Actes del XVII Congrés d'Història de la Corona ..., pp. 6-7, y «La historiografia de la cartoixa ...», Boletín de la Sociedad Castellonense de Cultura, LXXVIII, 2002, pp. 441-442.

${ }^{56}$ Ferrer Orts, A. op. cit., p. 89, «En torno a la actividad...», Actes del XVII Congrés d'Història de la Corona ..., p. 9, y «La historiografia de la cartoixa ...», Boletín de la Sociedad Castellonense de Cultura, LXXVIII, 2002, p. 457.

57 Ferrer Orts, A. «La historiografia de la cartoixa ...», Boletín de la Sociedad Castellonense de Cultura, LXXVIII, 2002, p. 437 .
}

$A E A$, LXXVIII, 2005, 309, pp. 71 a 81 


\begin{tabular}{|l|l|c|l|}
\hline \multicolumn{4}{|c|}{ CARTUJA DE ARA CHRISTI } \\
\hline IGLESIA (TRASALTAR ) & \multicolumn{2}{c|}{ PERÍODO (1630-1639) } \\
\hline \multicolumn{1}{|c|}{ ARTÍFICE } & \multicolumn{1}{c|}{ PROFESIÓN } & CRONOLOGÍA & \multicolumn{1}{c|}{ ACTUACIONES } \\
\hline RAFAEL MALLORQUÍ & Herrero & $1632-1639$ & Cúpula. \\
\hline MARTÍ D’ORINDA & Maestro de obras & $1632-1639$ & Mazoneria. \\
\hline PALLARÉS & Maestro de obras & $1631-1632$ & Mazoneria. \\
\hline JAUME REBULL & Maestro de obras & $1643-1647$ & Talla. \\
\hline JOAN BTA. TORMO & Escultor & $1651-1653(?)$ & Retablo. \\
\hline
\end{tabular}

Tabla 3

\begin{tabular}{|l|l|c|l|}
\hline \multicolumn{5}{|c|}{ CARTUJA DE ARA CHRISTI } \\
\hline \multicolumn{1}{|c|}{ SACRISTÍA } & \multicolumn{2}{c|}{ PERÍODO (1631-1633) } \\
\hline \multicolumn{1}{|c|}{ ARTÍFICE } & \multicolumn{1}{c|}{ PROFESIÓN } & CRONOLOGÍA & \multicolumn{1}{c|}{ ACTUACIONES } \\
\hline ANTONI BADENES & Maestro de obras & $1631-1634$ & \\
\hline A. BADENES ( hno. de ) & Peón (?) & $1631-1632$ & \\
\hline AGUSTÍ CUEVAS & Carpintero & $1662-1665(?)$ & Cajones. \\
\hline PERE JOAN ESPASA & Carpintero & $1657-1662(?)$ & Cajones. \\
\hline VICENT GAZO & Carpintero & $1662-1665(?)$ & Cajones. \\
\hline ANTONI MIRÓ & Maestro de obras & 1640 & \\
\hline FRA ANTONI ORTIZ & Maestro de obras & 1630 & Supervisor. \\
\hline BERNAT RIBAULO & Carpintero & $1662-1665(?)$ & Cajones. \\
\hline MOSSÉN GUILLEM ROCA & Maestro de obras & $1625-1631$ & \\
\hline FRANCESC SAYES & Maestro de obras & 1631 & Visura. \\
\hline JOAN BTA. TORMO & Escultor & $1651-1653(?)$ & Retablo. \\
\hline JOAN BTA. VERGARA & Carpintero & & Puerta. \\
\hline GUILLEM VILA & Maestro de obras & 1633 & Visura. \\
\hline
\end{tabular}

Tabla 4

\begin{tabular}{|l|l|c|l|}
\hline \multicolumn{4}{|c|}{ CARTUJA DE ARA CHRISTI } \\
\hline IGLESIA (ESPADAÑA ) & \multicolumn{2}{|c|}{ PERÍODO (1639-1640) } \\
\hline \multicolumn{1}{|c|}{ ARTÍFICE } & PROFESIÓN & CRONOLOGÍA & ACTUACIONES \\
\hline FRANCESC CERDÁ & Herrero & 1640 & Campana mayor. \\
\hline VICENT DOMÍNGUEZ & Carpintero & 1640 & Campana mayor. \\
\hline
\end{tabular}

Tabla 5

nado profesionalmente con el cenobio entre 1636-1657, creemos que trabajó como tapiador en el imafronte de la iglesia ${ }^{58}$. En marzo de 1640 el herrero Francesc Cerdà suministró metales para proceder a la fundición de la campana mayor de la espadaña ${ }^{59}$, tarea que concluyó el campanero Joan Vidal en mayo del citado año ${ }^{60}$.

Después de la bendición del templo por el arzobispo Aliaga, el 16 de noviembre de $1640{ }^{61}$, los trabajos de acondicionamiento continuaron, si bien referidos a obras de ornato, como el esgrafiado del intradós de la cúpula ${ }^{62}$ o la elaboración de retablos ${ }^{63}$, pinturas ${ }^{64}, \ldots$

${ }^{58}$ Ferrer Orts, A. «En torno a la actividad ...», Actes del XVII Congrés d'Història de la Corona ..., p. 7, y «La historiografia de la cartoixa ...», Boletín de la Sociedad Castellonense de Cultura, LXXVIII, 2002, p. 449.

${ }^{59}$ Ferrer Orts, A. «La historiografia de la cartoixa ...», Boletín de la Sociedad Castellonense de Cultura, LXXVIII, 2002, p. 424.

${ }^{60}$ Ferrer Orts, A. Op. cit., pp. 84 y 89 y «La historiografia de la cartoixa ...», Boletín de la Sociedad Castellonense de Cultura, LXXVIII, 2002, p. 458.

61 A.H.N., Códices, 1.372 B, f. 107 r.v.

${ }^{62}$ Ferrer Orts, A. op. cit., p. 102 y «Presencia de la decoración esgrafiada en la arquitectura valenciana (1642-1710)», Boletín del Museo e Instituto 'Camón Aznar', LXXXIV, 2001, p. 39. Como señalamos en la nota 54, los esgrafiados del cimborrio y cúpula del templo cartujano son los ejemplares más antiguos que hemos datado en tierras valencianas (ca. 1642), circunstancia que lo convierte en el precursor de esta modalidad decorativa en el citado territorio.

${ }^{63}$ Ferrer Orts, A. op. cit., pp. 84, 85 y 90, «En torno a la actividad ...», Actes del XVII Congrés d'Història de la Corona ..., p. 9, y «La historiografia de la cartoixa ...», Boletín de la Sociedad Castellonense de Cultura, LXXVIII, 2002 , p. $456-457$.

${ }^{64}$ Ferrer Orts, A. op. cit., pp. 85 y 90 o «La historiografia de la cartoixa ...», pp. 431 y 434.

$A E A$, LXXVIII, 2005, 309, pp. 71 a 81 
Con todo, los desperfectos ocasionados por otra tormenta hicieron que interviniera en la reparación de la calota Jaume Rebull, maestro de obras de la vecina Rafelbunyol, a partir de agosto de $1645^{65}$. Situación que se repetirá casi treinta años después, esta vez remediada con la participación de los maestros de obras Joan Claramunt y Francesc Padilla ${ }^{66}$.

A modo de conclusión, podemos decir que la cúpula de Ara Christi nació al mismo tiempo que se planificaba la iglesia, ésto es, el 17 de enero de 1620 — eso sí, con serias dudas sobre su correcta construcción, pues sólo la intervención de Roca parece asegurar su futura erección-. Así, el 6 de febrero del año siguiente se señalaron los cimientos del complejo, a la vez que se redactaron ocho cláusulas referidas a la iglesia y su cúpula, pues «[...] se advierte que no se aparten en ninguna manera de la traça y modelo questá hecho para dicha fábrica $[\ldots] \gg{ }^{67}$. Más tarde, el 4 de marzo se procedió a la colocación de la primera piedra, con lo que comenzó la edificación de nueva planta de la cartuja, así como de la cúpula de su iglesia mayor,finalizada durante el transcurso de 1631, si bien el templo quedaría resuelto, excepto su exornación, en las postrimerías de $1640^{68}$.

\footnotetext{
${ }^{65}$ Barlés Báguena, E. art. cit., p. 341 y Ferrer Orts, A. op. cit., p. 89, «En torno a la actividad ...», Actes del XVII Congrés d'Història de la Corona ..., p. 7, y «La historiografia de la cartoixa ...», Boletín de la Sociedad Castellonense de Cultura, LXXVIII, 2002, p. 448-449.

${ }^{66}$ A.R.V., Clero, libro $\mathrm{n}^{\circ} 2.951$, f. 14 r.v. y 15 r. Sobre sus perfiles profesionales véase Ferrer Orts, A. «En torno a la actividad ...», Actes del XVII Congrés d'Història de la Corona d'Aragó ..., p. 5 y 7, y «La historiografia de la cartoixa ...», Boletín de la Sociedad Castellonense de Cultura, LXXVIII, 2002, pp. 424-425 y 444-445.

${ }^{67}$ Ferrer Orts, A. «En torno a la actividad ...», Actes del XVII Congrés d'Història de la Corona ..., p. 13.

${ }^{68}$ Procede señalar que el féretro con los restos de doña Elena Roig y de Artés fue trasladado al cenobio el 2 de julio de 1656 por voluntad del prior Joan B. Giner "[ ... ] i el posaren darere lo sacrari, al matex peu de la llàntia [ ... ]» (o sea, quedó ubicado en el trasagrario).
} 